\title{
CAPTURING OF UNEVEN DEFORMATIONS OF LIGHTENED 3D PRINTED PARTS
}

Filip Klejch ${ }^{1 *}$, Eva Schmidová1, Jakub Vágner², Ivana Zetková ${ }^{3}$

\begin{abstract}
This paper deals with the problematics of tensile testing of maraging steel lightweight cylindrical samples with internal structures, intended for automotive application. The samples were made with DMLS technology and tensile tested under static strain rate of $0.002 \mathrm{~s}^{-1}$. During the loading, uneven deformations and multiple necking occurred on the samples. The mentioned effect significantly affects the state of stress and deformation at the final fracture zone, as well as the total deformation into the fracture, that means as a matter of fact it distorts the standard tensile test record. Therefore, a methodology for capturing the deformation response using the ARAMIS optical system was proposed. The methodology is presented and verified by set of experiments for BCC internal structure.
\end{abstract}

Keywords 3 3D printing of metal, Additive Manufacturing, DMLS, Maraging steel MS1, ARAMIS GOM 4M, tensile test, uneven deformation capturing methodology

\section{INTRODUCTION}

Most of current development and research in the automotive field is devoted to vehicle weight savings and electromobility. This trend came up automatically because of the tightening of environmental protection, especially of considerable emphasis on lower emissions from internal combustion engines. One of the possibilities to save vehicle weight is to use 3D printing (additive manufacturing) technologies to produce special lightweight components. This weight loss can be achieved by suitable material removal using the so-called topological optimization, or by suitable use of internal structures. The greatest weight loss can be achieved on the metallic materials of vehicles, so the development and research around 3D metal printing technologies is very intensive. (Milewski, 2017)

The initiation of the use of metal 3D printing in production requires considerable research into material properties, which may differ from commonly used materials. As an example may be a different microstructure of steel or anisotropy of mechanical properties in different directions of loading/testing, see (Schmidova et al., 2019). Also, subsequently when using internal structures designed to lighten the part while maintaining good functionality, we can get to the solution of unusual material testing tasks. An example is a common tensile test on cylindrical specimens with internal structures, where we encounter a problem in evaluating the results and capturing non-uniform deformations, which is the main subject of this paper. The solution may be to use the ARAMIS 4M optical measuring system from GOM GmbH, as will be presented.

\footnotetext{
${ }^{1}$ University of Pardubice, Faculty of Transport Engineering, Department of Mechanics, Materials and Machine Parts, Studentska 95, 532 10, Pardubice

${ }^{2}$ University of Pardubice, Faculty of Transport Engineering, Department of Transport Means and Diagnostics, Studentska 95, 532 10, Pardubice

${ }^{3}$ University of West Bohemia, Faculty of Mechanical Engineering, Department of Machining Technology, Univerzitni 22, 301 00, Pilsen

* corresponding author, phone: +420 466038 923, e-mail: filip.klejch@upce.cz
} 
Main technologies used for 3D metal printing are PBF (Powder Bed Fusion) and DED (Direct Energy Deposition) technologies. Used materials are in the form of fine metal powder or, in the case of DED, they can also be in the form of metal wire (Bourell et al., 2017). Further attention is paid in particular materials that are used in the form of a fine metal powder. There exist about 40 available materials that represent a relatively wide range of different specific properties and use. But the disadvantage may be a higher price and the absence of conventional cheaper steels. Commonly used are stainless and tool steels, titanium alloys, aluminium alloys, cobalt alloys, copper alloys, nickel superalloys and precious metals (Metal AM, 2018). Tested samples were made of high-strength Maraging steel using the DMLS production method. Direct Metal Laser Sintering technology (DMLS) can be considered the most used method for the production of complex parts.

This paper describes a method of capturing non-uniform deformations in tensile tests of 3D printed cylindrical samples containing internal structures.

\section{MATERIAL AND MEASURING SYSTEM}

When performing tensile tests of lightened cylindrical samples made of high-strength steel, there appeared uneven surface deformations and multiple necking, resp. heterogeneous plasticity. Mentioned deformations were influenced by the type and arrangement of internal structures. In the next text, the used material, internal structures and the main capturing tool are introduced.

\subsection{Maraging Steel MS1}

Tested samples were made using DMLS method from Maraging steel material grade of MS1. MS1 is a metal powder specially developed for powder bed fusion processes and its composition corresponds to steel 1.2709 (X3NiCoMoTi 18-9-5). The chemical composition of the tested steel in comparison with the composition of conventional Maraging steel is shown in Tab. 1. The characteristics and use of this steel are similar to conventional Maraging steels. The parts have good machinability and hardenability after the printing process - it is possible to reach hardness of more than $50 \mathrm{HRC}$ with postprocess age-hardening. That means a precipitation hardening, which makes steel achieve enormous strength. But tested samples were only after the primary stage of production, that means without hardening to determine the effect of the used technology, including the natural surface relief (Schmidova et al., 2019). Only solution annealing heat treatment was applied: heating in a protective atmosphere to the austenitization temperature was applied - the endurance at this temperature was 1 hour, then followed by rapid cooling below $500{ }^{\circ} \mathrm{C}$, followed by cooling on air.

Tab. 1 Chemical composition of MS1 in comparison to 1.2709

\begin{tabular}{|c|c|c|c|c|c|c|c|c|c|c|c|}
\hline & C & Si & Mn & $\mathbf{P}$ & $S$ & $\mathrm{Cr}$ & Mo & $\mathrm{Ni}$ & Co & $\mathbf{T i}$ & Al \\
\hline MS1 & $\leq 0.03$ & $\leq 0.1$ & $\leq 0.1$ & $\leq 0.01$ & $\leq 0.01$ & $\leq 0.5$ & 4.5 & 17.0 & 8.5 & 0.6 & 0.05 \\
\hline & & & & & & & 5.2 & 19.0 & 9.5 & 0.8 & 0.15 \\
\hline 1.2709 & $\leq 0.03$ & $\leq 0.1$ & $\leq 0.15$ & $\leq 0.01$ & $\leq 0.01$ & $\leq 0.25$ & $\begin{array}{l}4.5 \\
5.2\end{array}$ & $\begin{array}{l}17.0 \\
19.0\end{array}$ & $\begin{array}{l}8.5 \\
10\end{array}$ & $\begin{array}{l}0.8 \\
1.2\end{array}$ & - \\
\hline
\end{tabular}

Tested samples were cylindrical with lightened internal structures and the testing was focused on the mechanical response of the material. The strain rate was determined at the beginning of the tests relative to the deformation part of the sample. Based on the EN 10002-1 standard, the strain rate was set at $0.002 s^{-1}$ - static tensile test. The samples were hollow with two variants of internal structures - gyroid and cubic (BCC), the external dimensions were identical (see Fig. 1). Printing direction is indicated by an arrow. 


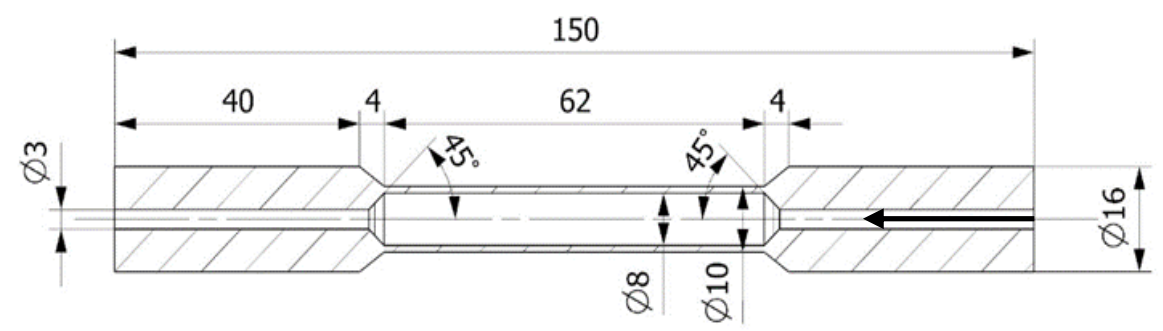

Fig. 1 Sample dimensions (Purš et al., 2019)

Two types of internal structures were used. The main meaning of the use of these structures is weight loss or reinforcement of the components. The principle is to copy the basic cell in space, which leads to the formation of large structures. The basic cells are shown in the Fig. 2 on the left, in the middle is a graphic of the copied cells in the sample and on the right are views inside to the broken samples. The length of the basic cell edge was $5 \mathrm{~mm}$ for all samples, another indicator was the volume fraction of mass in the basic cell, which was $13.5 \%$ (that means the cell volume was filled with a mass of 13.5\%). (Purš et al., 2019) These are the two most used types of internal structures, which are technologically advantageous for use in internal spaces - they do not need to build support structures during the 3D printing process.

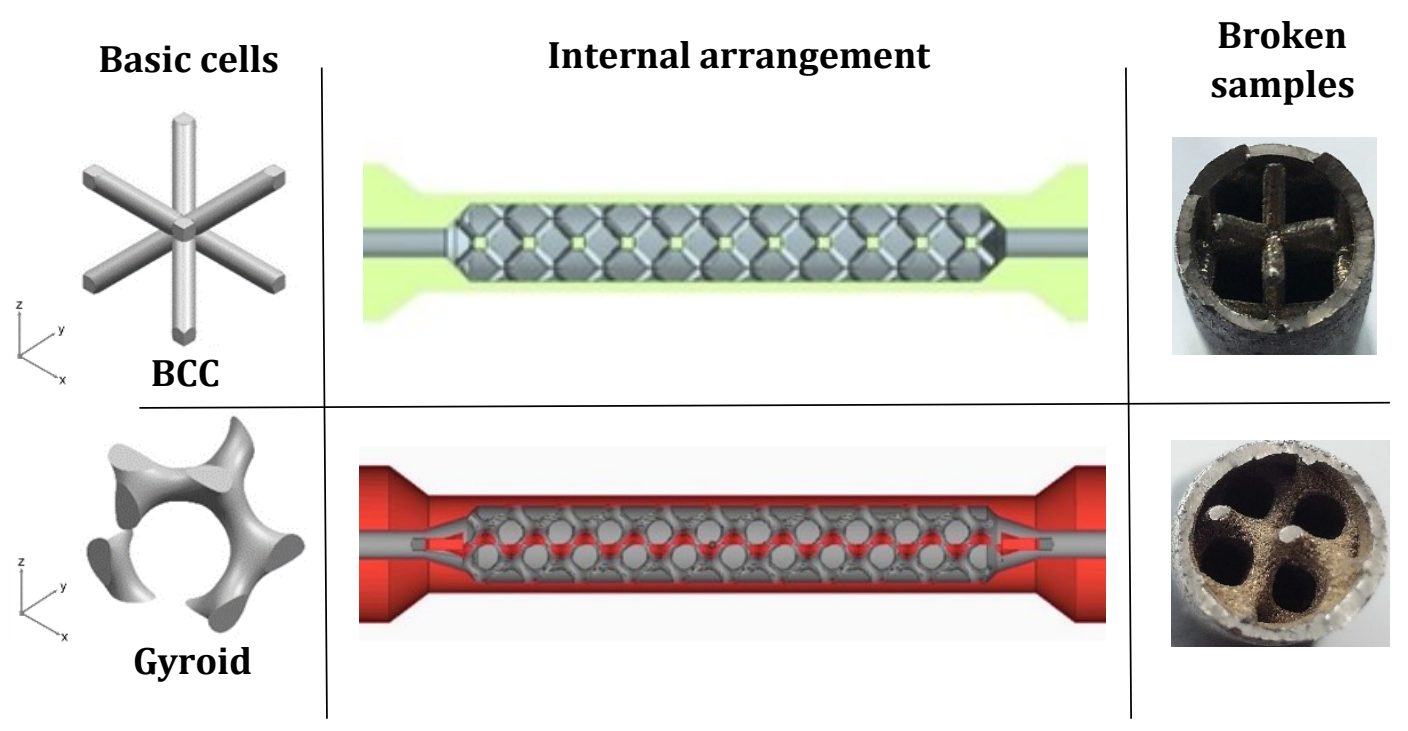

Fig. 2 Introduction of internal structures (Purš et al., 2019)

\subsection{Measuring system ARAMIS 4M}

Non-contact optical 3D deformation measuring system ARAMIS GOM 4M was used to continuously capture the formation of heterogeneous surface deformations, resp. stress - strain condition.

In the $4 \mathrm{M}$ version, the system is equipped with a sensor with two cameras and a light unit, which is placed on a camera support. The sensor is controlled by a powerful computer system, which is located in a mobile box - the whole system is therefore relatively easy to manipulate. The principle of function is that ARAMIS uses a sensor to take time-graded images of the scanned object (the first image is without deformation and the following are taken after or during deformation of the object) - the result is a series of images. The system can recognize the surface structure on the digital image from the cameras and allocate to it coordinates, resp. allocates coordinates to the image pixels. Subsequently, the system compares successive digital images and calculates the displacement and appearing deformations, which are then displayed on special graphics. If the surface of the sample does not have a sufficiently detectable surface structure, the 
object must be provided with a suitable color sample, for example stochastic color spray pattern. Measuring can be performed under static or dynamic loads. (ARAMIS manual, 2007)

Before each new measurement series, the system must be calibrated to the measured objects. With the sensor, it is possible to measure small and large objects (from $1 \mathrm{~mm}$ to $2000 \mathrm{~mm}$ ) with deformations from $0.01 \%$ up to several hundred percent. This means that the sensor unit must be suitably positioned with a camera support to sense the required measured volume on the object. Then all the pictures are taken. Calculation of the project depends on the definition of the monitored area (so-called computation mask), which the user defines in the program, and defining of the starting point. During the calculation, the system monitors the deformation of the object on the images using the so-called facets (square or rectangular sections of the images - e.g. 15x15 pixels). The size of the facets in pixels can be set at the beginning of measuring. The system combines images from both sensor cameras and uses the photogrammetric method to determine the 3D coordinate of each facet. The measurement result is available as a 3D preview. The program also has the ability to create reports and export measured data. Some possible outputs of the program are shown in the section 3. (ARAMIS manual, 2007)

Fig. 3 shows the setup of a static tensile test. The left part of the figure shows a sample clamping and the setup of the ARAMIS sensor. The right part of the figure shows a detail of clamped sample with placed extensometer for measuring the longitudinal elongation. A stochastic paint pattern can be seen on the surface of the sample. The Inova Test Control system was used to control the course of the test.

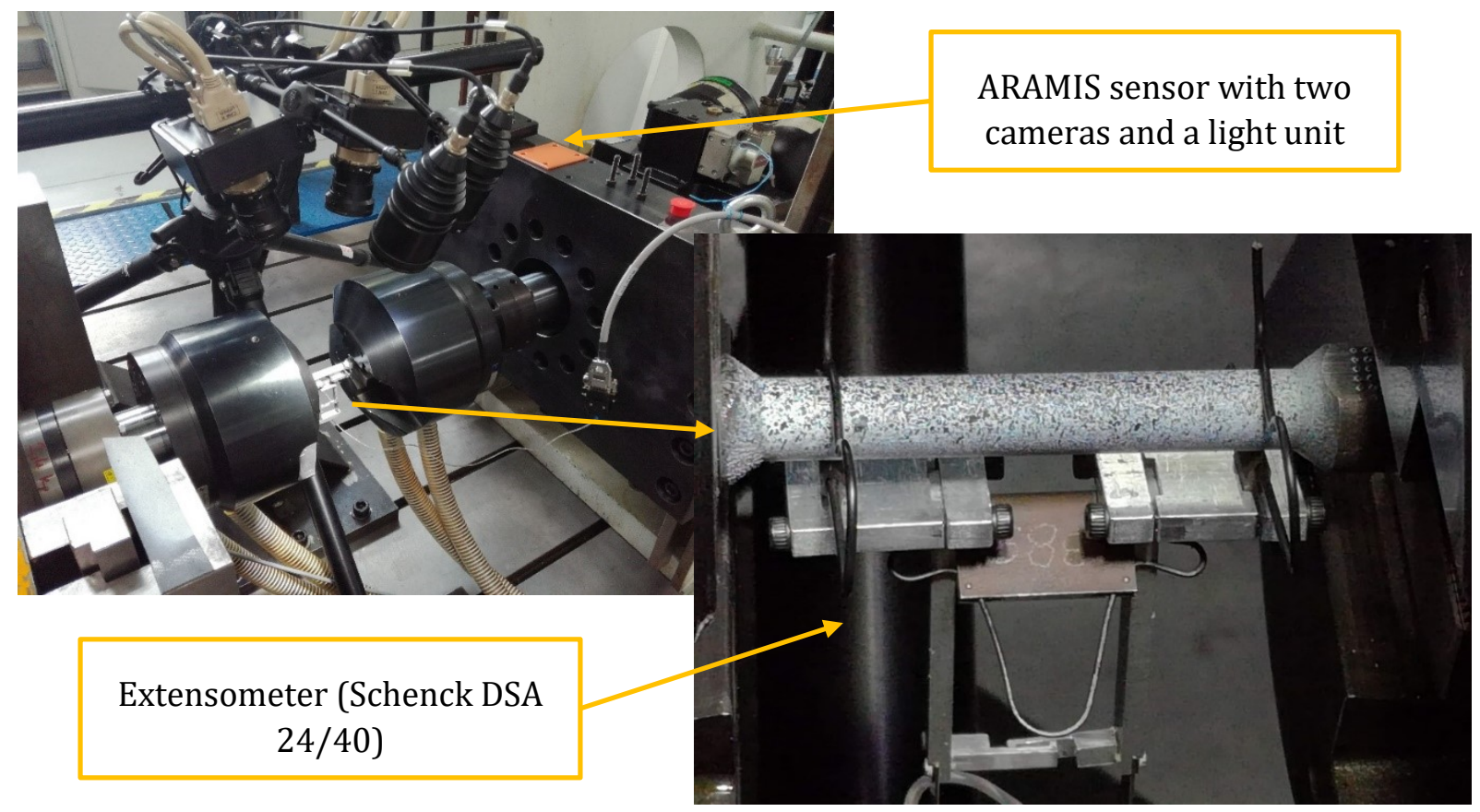

Fig. 3 Setup of a static tensile test

\section{PROPOSED METHODOLOGY, RESULTS OF MEASUREMENT}

During the testing appeared multiple necking of the samples. This multiple necking, in a certain part of the loading, became more noticeable in the fracture zone of the sample. The necking was visible to the naked eye on the damaged samples, and it is clear that there was a heterogeneous plasticity development. This is confirmed by the unevenness of surface deformations, which, like the zones of necking, depends on the geometry of the internal structures. Multiple necking and uneven surface deformations differ for individual types of structures (BCC x Gyroid). The mentioned effect significantly affects the state of stress and deformation at the final fracture zone, as well as the total deformation into the fracture, that means as a matter of fact it distorts the standard tensile test record. That means this phenomenon had to be captured 
and monitored so the obtained results were correct. Therefore, a procedure for evaluating the deformation response using the ARAMIS optical system was proposed. A methodology for the evaluation of the subject cylindrical samples was developed in the ARAMIS and MS Excel software. The methodology is further presented on one reference sample with BCC internal structure.

\subsection{Reference BCC sample}

The BCC sample showed a slightly larger unevenness of deformations, so the methodology is presented using this type of internal structure. The next text presents the outputs from the ARAMIS system (note: all images are taken with the left camera of the 3D sensor).

Fig. 4 shows a clamped specimen during the measurement. The area highlighted in green is the so-called computation mask, which is the area where the displacement calculation is performed. The points marked in red are the starting points that must be determined to perform the calculation. The image is taken at stage 460, which means 46 seconds from the start of the measurement and about 0.2 seconds before the sample breaks.

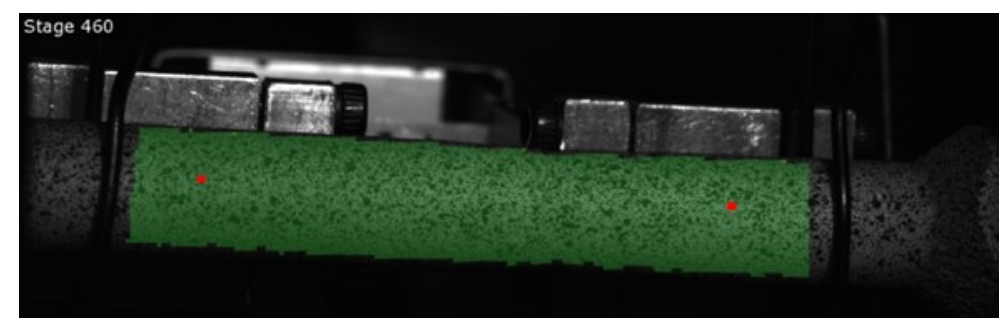

Fig. 4 Computation mask

Fig. 5 shows the already performed calculation for stage 230, that means at a time of 23 seconds from the beginning of the measurement. The epsilon $\mathrm{x}$ in $\%$ is shown here. This illustration suitably shows the distribution of deformations on the surface of the sample, the influence of the internal structure on the deformation is obvious. Stage 230 is shown here because approximately at this time a more significant deformation occurs around the fracture zone (red areas).

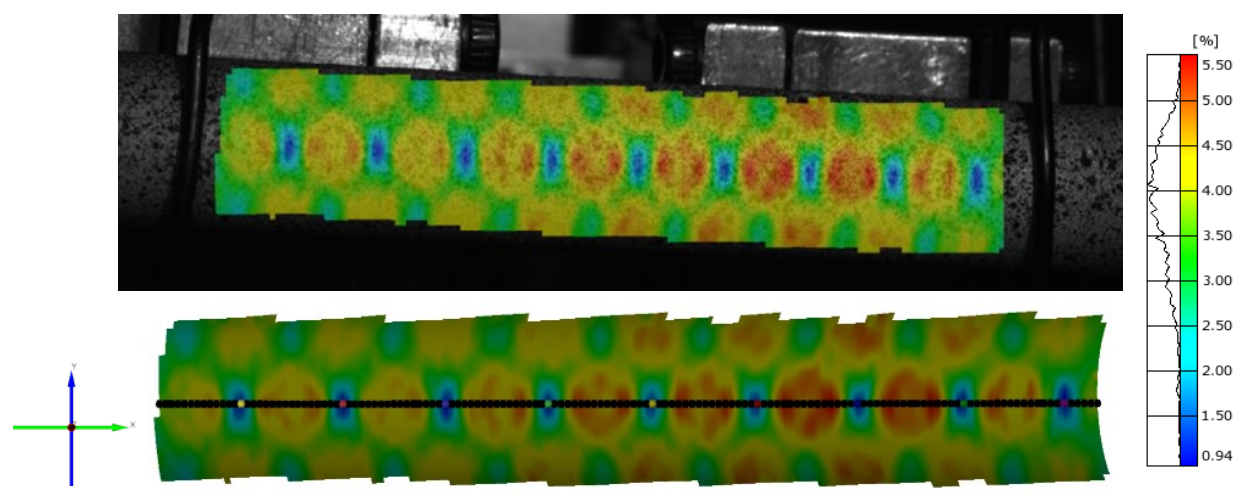

Fig. 5 Epsilon $x$ in stage 230 (BCC)

For reproducibility of capture and expression of deformations along the length of the evaluated area, it was necessary to define a reference line of points (section) in the axis of the measured sample. The number of points in the line is limited by the number of elements (facets) from which the computation mask is assembled. The section is shown at the bottom of Fig. 5, where points in the local deformation minimums and edges of the computation mask are also highlighted. These points were used for the evaluation, which will be described later.

Using the given line of points, it is possible to create a graph that shows the epsilon $\mathrm{x}(\%)$ along the length of the line - the so-called Multi-Section diagram. Fig. 6 shows this dependence for stage 230 - it can be seen 
from the graph that the deformations begin to locate in the area of the fracture, where it gradually begins to necking (the area marked in green).

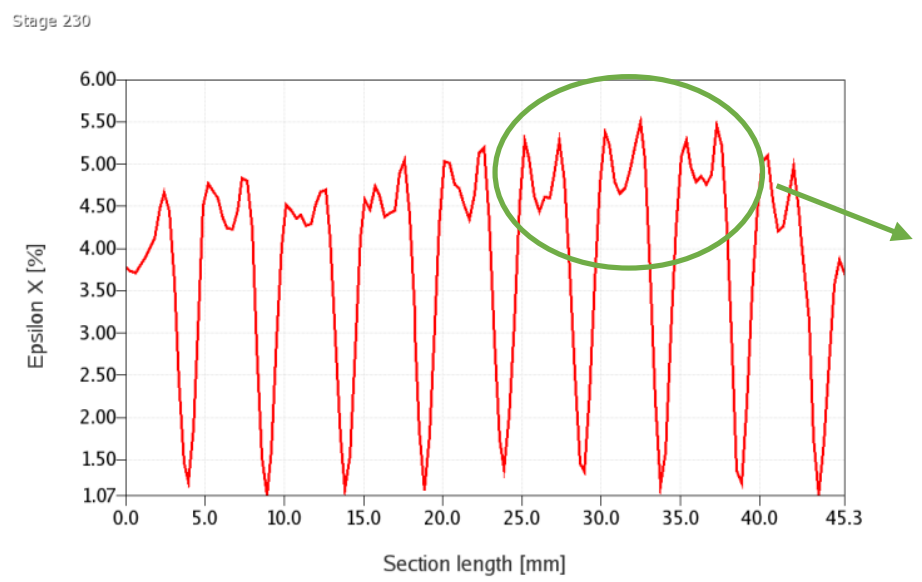

Area of the fracture and
multiple necking. The marked
peaks of surface
deformations begin to
predominate over the
surrounding ones.

Fig. 6 Multi-Section diagram for stage 230 (BCC)

This elongation can be also expressed appropriately graded for different time points (stage) in a MultiStage-Section diagram. Fig. 7 shows the time graded curves from stage 230 to stage 460 .

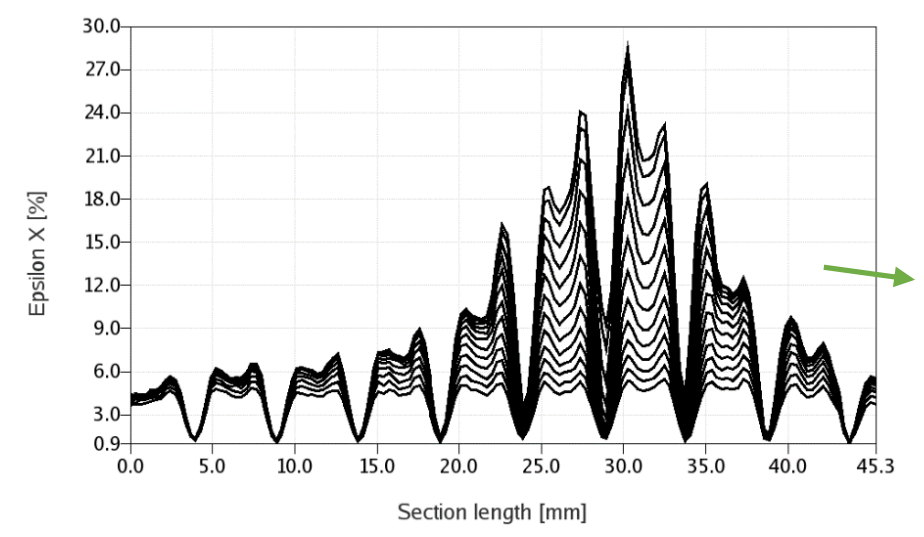

Graph shows the development of surface deformations over time. The lower curve shows stage 230 (time $23 \mathrm{~s}$ ) and the upper curve stage 460 (time 46 s), i.e. just before sample failure. The graph shows the development and localization of deformation over time loading.

Fig. 7 Multi-Stage-Section diagram (stage 230-460), BCC

Epsilon $x$ corresponding to stage 460 is shown in Fig. 8. These are the surface deformations just before the failure of the sample (approx. $0.2 \mathrm{~s}$ before the failure). In the upper part of the figure you can see a mesh of facets (elements) with local coordinate systems, which are oriented parallel to the section line (in the direction of the x-axis). The lower part of the figure shows the rotation of the surface of the results, the area of fracture formation is obvious - it is possible to see necking on the wavy part of section

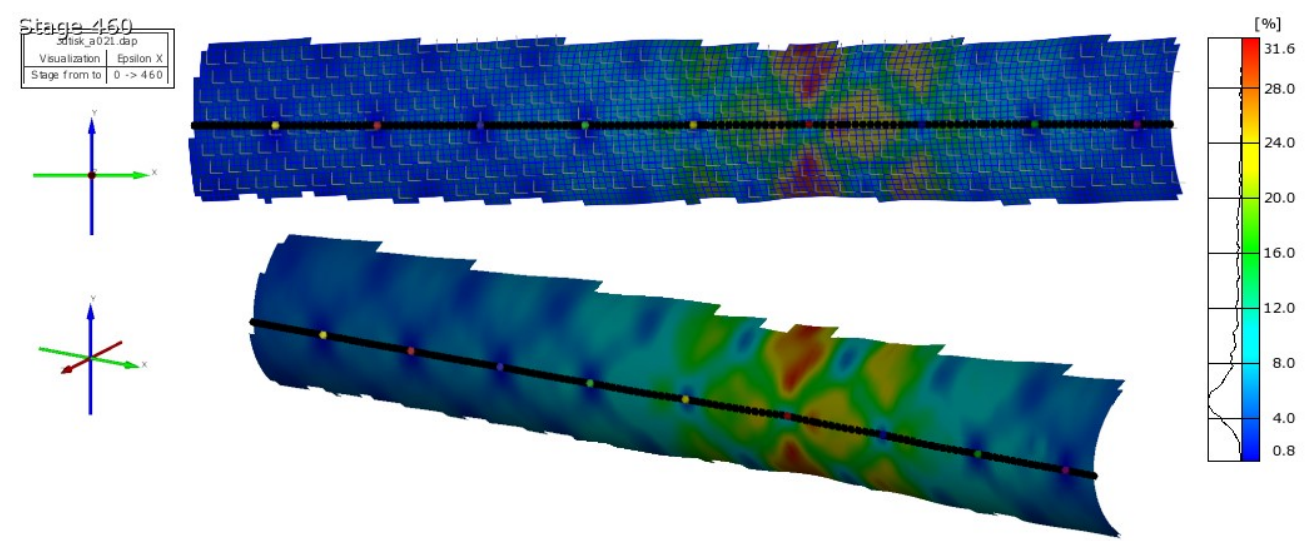

Fig. 8 Epsilon $\mathrm{x}$ in stage 460 (BCC) 
For further evaluation, the Multi - Section diagram for stage 460 was divided into 10 parts, which are shown in Fig. 9. The parts are bounded by local minimums of epsilon $\mathrm{x}$ - these minimums are marked in the previous figures (Fig. 5 and 8) by colored dots on the section line, the endpoints are given by the edge of the computation mask.

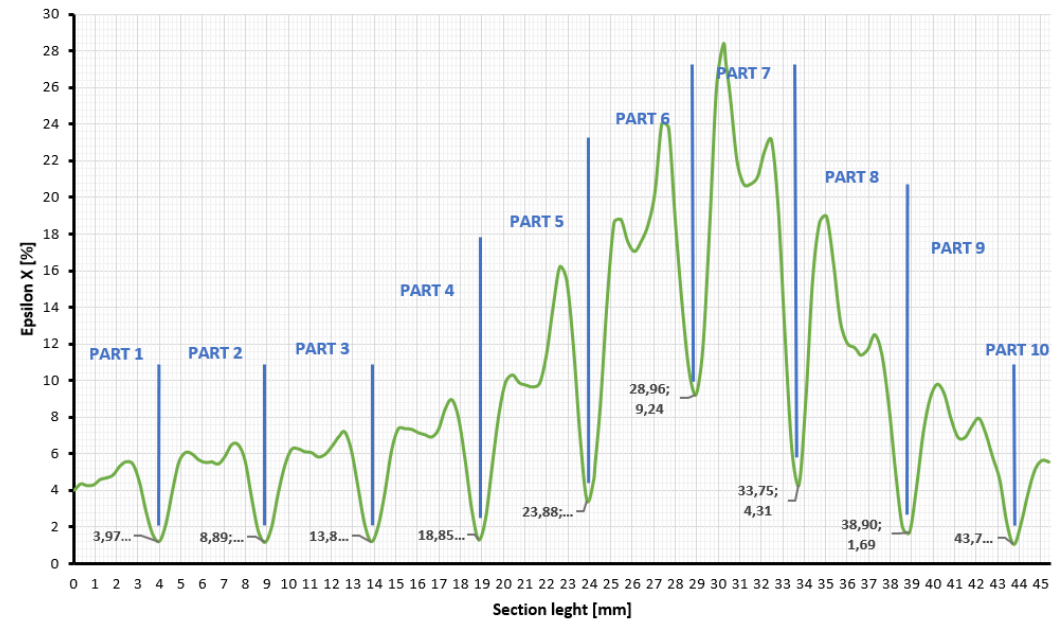

Fig. 9 Multi-Section divided diagram for stage 460 (BCC)

The ARAMIS system can, similarly to epsilon $\mathrm{x}$, show the displacement of individual points. However, this graphical representation is only relevant for reading the extension of the entire computation area. Therefore, the given parts in Fig. 9 were selected and the extension of these individual areas was calculated. Knowing the load force over time, it was possible to draw tensile diagrams for selected parts, see Fig. 10. The size of the extension of individual parts is clear from the graph. The graph also shows a tensile diagram from the INOVA device on which the sample was tested.

The cumulative elongation, the elongation from the used extensometer, and the elongation subtracted from the ARAMIS graphic differs only in the order of hundredths of a millimetre. This confirms the correct measurement setting. Another important thing is that the force on the yield strength is the same for all calculated tensile curves and corresponds with the force on the yield strength of the INOVA tensile curve. This confirms the capability of the methodology used to detect the localized plasticity, and to capture the local differences in the propagation of plasticity up to the final fracture. This is closely related to the 3D printing technology itself and it is important to monitor the local behaviour of this type of material under loading.

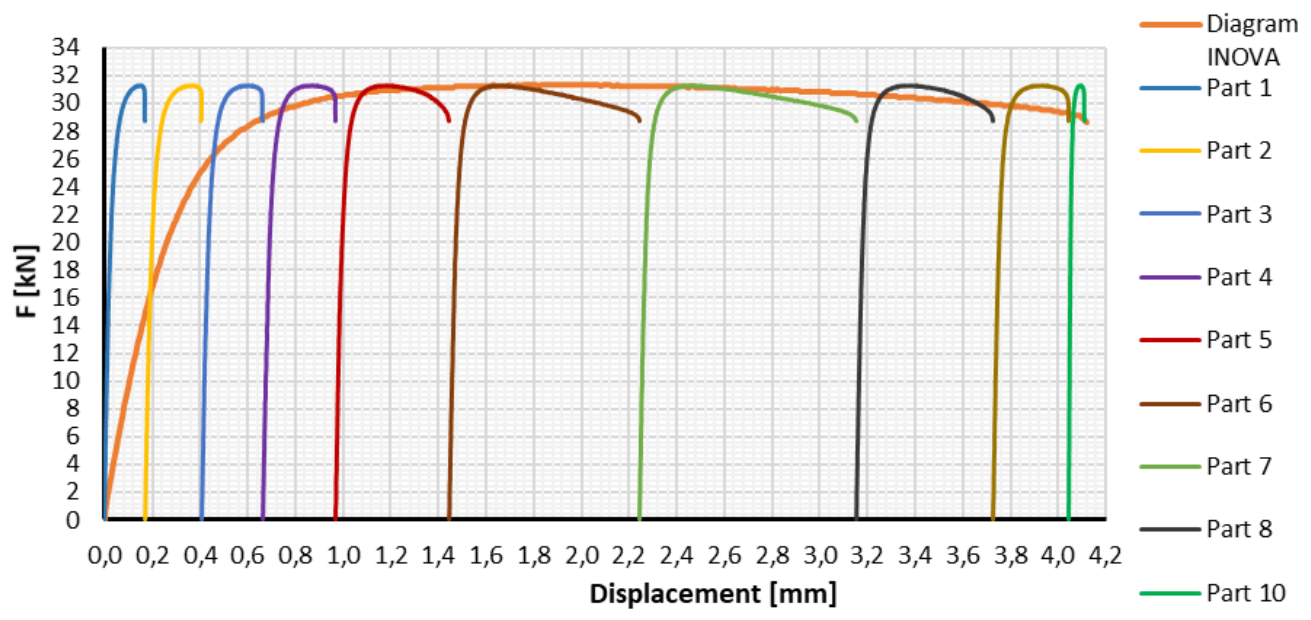

Fig. 10 Cumulative elongation (BCC)

One disadvantage, which results from the principle of the ARAMIS system, is the limitation to surface sensing only, and thus to the capturing of only surface deformations. 


\section{CONCLUSIONS}

Using the presented methodology, using the optical system ARAMIS and the quantitative evaluation of additional points displacements, it is possible to very accurately evaluate the unevenness of surface deformations. The standard approach to the evaluation of inhomogeneous deformation of lattice structures is based on the pressure tests. In case the samples without any shell are used, i.e., pure lattice structures are tested without any influence of shell to core connection. The tests allow us to determine the elasticity characteristics and strain hardening characteristics commonly used for topology optimization, especially when using numerical methods. Firsov et al. (Firsov, D.G. et al., 2020) introduced the deformation response of the various lattice structures created by 3D, selective laser melting technology. The fields of transverse, longitudinal, and shear deformation were constructed using the digital image correlation method serving for evaluation of heterogeneity in the stress field distribution. Because the perimeter layer was missing, the shell vs. core interface could not be included in the consideration. DIC methods is also useful for the detection of inhomogeneous deformation propagation at the crack tip, as information about material resistance to internal imperfections (Matsumoto, R., 2013), or as a validation method for acoustic emission (Murasawa et al., 2014).

As was found during the tests performed, the shell vs. lattice core transition is one of the most important constraints, and thus the important criterion for lightweight 3D printed products. The used testing approach was therefore based on the full-latticed round bar samples design, intended for uniaxial tests. The accuracy of the method follows from the cumulative elongation graph, where the individual methods of determining the total elongation are almost the same. This procedure can therefore be used to independently locate the deformation, including the limit deformation, leading to the destruction of the sample.

Another thing that follows from the methodology is the importance of monitoring the local behaviour of this 3D printed material under loading.

\section{Acknowledgements}

This research has been realized using the support of Technological Agency, Czech Republic, programme National Competence Centres, project \# TN01000026 Josef Bozek National Center of Competence for Surface Vehicles and project FV30149 MPO-TRIO.

This support is gratefully acknowledged.

\section{References}

ARAMIS, User manual - Software, GOM mbH, Braunschweig, 2007

Bourell, D., Kruth J.P., Leu M., Levy, G., Rosen, D., Beese, A. M. and Clare, A. 2017. Materials for additive manufacturing. CIRP Annals - Manufacturing Technology, 66(2), pp. 359-681 (23). DOI:

https://doi.org/10.1016/j.cirp.2017.05.0097

Firsov, D.G., Konev, S.D., Dubinin, O.N., Evlashin, S.A., and Shishkovsky, I.V., 2020. Localization of Deformation in Lattice Structures of 3D-Printed Samples of 03X17H14M2 Steel. Technical Physics Letters, 46(7), pp.683-686

Inovar Communications Ltd. - Metal AM. (C) 2018. Metal powders - the raw materials. [Online]. Available at: https://www.metal-am.com/introduction-to-metal-additive-manufacturing-and-3d-printing/metalpowders-the-raw-materials/ [Accessed: 02. 10. 2020].

Matsumoto, R., Kubota, M. and Miyazaki, N., 2016. Development of deformation measurement system consisting of high-speed camera and digital image correlation, and its application to the measurement of large inhomogeneous deformations around the crack tip. Experimental Techniques, 40(1), pp.91-100. 
Milewski, John 0. 2017. Additive Manufacturing of Metals: From Fundamental Technology to Rocket Nozzles, Medical Implants and Custom Jewelry. Cham, Schwitzerland: Springer International Publishing. ISBN 9783-319-58205-4. [online]. Available at: https://books.google.cz/books?id=XlsqDwAAQBAJ\&lpg=PP1\&dq=metal\%20additive\%20manufacturing $\underline{\text { \&pg }=\mathrm{PA} 8 \# \mathrm{v}=\text { onepage } \& \mathrm{q} \& \mathrm{f}=\text { false }}$ [Accessed: 02.10.2020].

Murasawa, G., Takahashi, R., Morimoto, T. and Yoneyama, S., 2015. Inhomogeneous deformation twinning measurement using digital image correlation and acoustic emission. Experimental Mechanics, 55(1), pp.6576.

Purš, H., Zetek, M., Schmidová, E.: 3D printing of computationally optimized metal parts using DMLS technology. Annual report on the solution of the project in the program TRIO ev. No. FV30149 in 2019

Schmidova E., Hojka P., Culek B. st., Klejch F. and Schmid M. 2019. Dynamic Strength and Anisotropy of DMLS Manufactured Maraging Steel. Communications - Scientific Letters of the University of Zilina, 21(3), pp. 35-39. Available at: http://komunikacie.uniza.sk/index.php/communications/article/view/1502 [Accessed: 02. 10. 2020]. 